\title{
INFRA-RED EMISSION FROM CLASSICAL NOVAE
}

\author{
Jim MacDonald \\ Department of Physics and Astronomy \\ University of Delaware, Newark, DE 19716, U.S.A.
}

\section{INTRODUCTION}

During the evolution of a classical nova outburst, there are four times at which a significant infra-red (IR) flux is expected. The first Isothermal Dust Phase has been observed in a number of novae and analyses of this phase give valuable information on the properties of the emitting dust. In order of time from visual maximum, the three later phases are due to IR emission from X-ray heated grains, fine-structure line emission from a cold phase of the nova nebula, and grain cooling from shocked gas at the interface between the nova ejecta and the interstellar medium. In the next sections, theoretical estimates of the peak IR luminosity, flux distribution and time of peak emission for each of these phases are discussed.

\section{THE ISOTHERMAL DUST PHASE}

The appearance of an IR excess in most slow and moderately fast classical novae some weeks after visual maximum is well documented. The peak IR luminosity, $\approx 10^{4} L_{\odot}$, indicates that the bulk of the luminosity from the stellar remnant is processed into IR photons during this phase. The onset of IR emission coincides with the visual transition phase, suggesting that the IR flux is due to radiation from dust grains condensed out of the nova ejecta. The IR photometric data is consistent with emission from optically thick dust. Dust temperatures have a common temporal behavior; exhibiting a decrease as IR maximum is approached, a rise following maximum and a fall at still later times. Typical dust temperatures at the time of maximum IR emission are $800-1000 \mathrm{~K}$ and hence the IR flux peaks at a wavelength of about 3 microns. However, the large IR luminosity implies such substantial fluxes at 12 and 25 microns that all galactic novae undergoing an isothermal dust phase should be detectable by an IRAS-like instrument.

\section{IR EMISSION FROM X-RAY HEATED GRAINS}

Hydrodynamic studies of nova eruptions predict that, following runaway, shell hydrogen burning of residual matter defines a phase of evolution characterized by constant bolometric luminosity, at a level compatible with the mass-luminosity relation for degenerate cores. During this constant bolometric luminosity phase, the photospheric temperature increases in response to reduction in envelope mass by nuclear burning and stellar mass loss.

The general features of the decline phase of novae lead to a prediction of the presence of a soft $\mathrm{X}$-ray phase about 1 to $10 \mathrm{yr}$ after visual maximum, just before cessation of nuclear burning in the 
stellar remnant. The flux distribution of soft X-rays detected by EXOSAT from Nova Muscae 1983, about 16 months after visual maximum, is compatible with a white dwarf remnant emitting $3.510^{5}$ $\mathrm{K}$ blackbody radiation at $10^{37} \mathrm{erg} / \mathrm{s}$.

Heating of grains by these soft X-rays will lead to a second phase of infra-red emission. The ratio of IR to X-ray luminosity is

$$
\frac{L_{I R}}{L_{X}} \approx 610^{-3}\left(\frac{M_{g}}{10^{-6} M_{\odot}}\right)\left(\frac{r}{10^{16} \mathrm{~cm}}\right)^{-2}
$$

where $M_{g}$ is the total mass of grains in the ejecta and $r$ is the distance of the dust shell from the central X-ray source. For a typical slow nova $L_{I R}$ will be about $100 L_{\odot}$. For moderately fast novae, $L_{I R}$ is expected to be less because the dust shell will be further from the central X-ray source. Using the emission efficiency for grains of pyrolitic carbon, the grain temperature is

$$
T_{g}=200\left(\frac{L_{I R}}{10^{2} L_{\odot}}\right)^{\frac{1}{5}}\left(\frac{M_{g}}{10^{-6} M_{\odot}}\right)^{-\frac{1}{5}} K
$$

and peak IR emission occurs at wavelength

$$
\lambda_{p k} \approx 12\left(\frac{T_{g}}{200 K}\right) \text { microns. }
$$

For $T_{g}=200 \mathrm{~K}$, it can be estimated that IR emission from X-ray heated grains should be detectable by an IRAS-like instrument for slow novae closer than about $4 \mathrm{kpc}$ and moderately fast novae closer than about $1 \mathrm{kpc}$.

\section{INFRA-RED FINE STRUCTURE LINE EMISSION FROM COLD NOVA NEBULAE.}

Photo-ionization models of the DQ Her nebula show that its low electron temperature is the result of the very high metal abundances that characterize the shell of DQ Her and other novae. For sufficiently low electron densities, infra-red fine structure lines, particularly the 52 and 88 micron lines of [O III], are very efficient coolants and low temperatures are achieved for a wide variety of radiation fields, including those expected from accretion onto white dwarfs in cataclysmic binaries. Since high metallicity is a feature of the majority of novae ejecta, most nova nebulae will undergo a cold phase when the electron density has dropped to below $\approx 10 \mathrm{~cm}^{-3}$.

In view of the large overabundances of neon found in the ejecta of a number of recent novae, the possibility arises that the 12.8 micron line from [Ne II] can also efficiently cool nova nebulae. Since the critical electron density for collisional de-excitation of this line is $\approx 10^{5} \mathrm{~cm}^{-3}$, neon-rich ejecta is expected to cool at an earlier phase of the nebular expansion than neon-poor ejecta. The stellar remnant may still be quite luminous and the heating of the nebula substantially larger than at later times. The neon fine structure line then will be a major contributor to the 12 micron flux. This point of view is strongly supported by the discovery of very strong 12.8 micron line emission in Nova Vulpeculae 1984 no. 2, 140 days after visual maximum. The total luminosity in this line was $\approx 80 L_{\odot}$ and hence the predicted 12 micron flux is comparable to that for the $\mathrm{X}$-ray heated grain model. Detection of significant 12 micron flux will place useful constraints on the photo-ionization model of cold nova shells and may help to set limits on the relative number of neon-rich to neon-poor novae, 
which has important implications for the theory of the formation of white dwarfs in cataclysmic binaries.

\section{INFRA-RED EMISSION FROM GRAIN COOLING OF SHOCKED GAS}

Classical novae eject a mass $M_{e j} \approx 10^{-4} M_{\odot}$ of material into the interstellar medium at speeds, $V_{e j}$, that range from $300 \mathrm{~km} / \mathrm{s}$ for slow novae to $1600 \mathrm{~km} / \mathrm{s}$ for very fast novae. The interaction of the ejecta with the ISM can be treated as a miniature supernova remnant. Initially the nova shell can be treated as a hard piston that drives a shock wave into the ISM with the shock wave preceding the nova shell. The pressure of the hot gas behind the shock slows the nova shell, bringing it to a stop when the mass of ISM material swept up by the shock is about $10 M_{e j}$. The high pressure of the post shock gas drives a reverse shock into the nova shell. Since the ejecta of many novae are rich in grains, grain cooling of nova shell material heated by the passage of the reverse shock can also produce large IR fluxes. For typical values of the thickness of the nova shell, $d$, the expected IR flux from grain cooling of the shocked gas is

$$
L_{I R} \approx 0.7\left(\frac{M_{e j}}{10^{-4} M_{\odot}}\right)\left(\frac{d}{10^{16} \mathrm{~cm}}\right)^{-1}\left(\frac{V_{\mathrm{rs}}}{300 \mathrm{~km} / \mathrm{s}}\right)^{3} L_{\odot} .
$$

where $V_{r s}$ is the speed of the reverse shock. If $n$ is the number density of nucleons in the ISM, the reverse shock forms at time $t_{r s}$ after visual maximum where

$$
t_{r s} \approx 700\left(\frac{M_{e j}}{10^{-4} M_{\odot}}\right)^{\frac{1}{3}}\left(\frac{n}{\mathrm{~cm}^{3}}\right)^{-\frac{1}{3}}\left(\frac{V_{e j}}{300 \mathrm{~km} / \mathrm{s}}\right)^{-1} \mathrm{yr} .
$$

Hence this form of IR emission will be detectable only from old remnants (age > $100 \mathrm{yr}$ ) of fast novae $\left(V_{e j}>800 \mathrm{~km} / \mathrm{s}\right)$. 